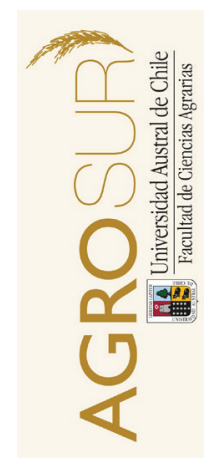

\title{
Variantes genéticas de beta caseína bovina: implicancia en la producción, características tecnológicas de la leche y la salud humana
}

\author{
Genetic variants of bovine beta casein: relationship with production, \\ milk technological traits and human health
}

\author{
Gatica, C. ${ }^{a, b}$, Alomar, D. ${ }^{c *}$ \\ ${ }^{a}$ Escuela de Graduados, Facultad de Ciencias Agrarias, Universidad Austral de Chile. Casilla 567, Valdivia, Chile. \\ ${ }^{b}$ CIA CENEREMA Facultad de Ciencias Veterinarias, Universidad Austral de Chile. Casilla 567, Valdivia, Chile.
}

${ }^{c}$ Instituto de Producción Animal, Facultad de Ciencias Agrarias, Universidad Austral de Chile. Casilla 567, Valdivia, Chile.

\begin{tabular}{l} 
A R T I C L E I N F O \\
\hline Article history: \\
Received 25.04.2018 \\
Accepted 02.08.2018 \\
\hline Keywords: \\
Caseins \\
Beta-casein \\
Kappa-casein \\
Genetic variants \\
Beta casomorphin 7 \\
\hline Review, \\
Food Processing and Quality \\
\hline${ }^{*}$ Corresponding author: \\
Daniel Alomar \\
E-mail address: \\
dalomar@uach.cl
\end{tabular}

\begin{abstract}
A B S T R A C T
Milk is a fundamental feed of all newborn mammals and a good source of proteins, fatty acids, micro elements, calcium, phosphorus, among others, for non-lactating humans. As world demand for foods increases, so does the concern and requirements of consumers for food quality and safety. In view of evidences of possible pathologies associated to milk consumption, detractors of the dairy industry have appeared, making use of these arguments. Research on the risks associated to milk ingestion has been conducted on the last twenty years and the existence of specific milk protein variants able to cause disorders such as milk intolerance, diabetes mellitus type 1, ischemic heart disease and food allergies, has been established. The determination of casein genetic variants and the association of the A1 beta-casein with human health disorders represent an opportunity, for the farmers to select replacements, retaining those cows with the desirable alleles, and also for the dairy industry to obtain safer dairy products. As a result, all the dairy supply chain, from producers to consumers, will be benefited.
\end{abstract}

\section{RESUMEN}

La leche es un alimento fundamental de todos los mamíferos luego del nacimiento y una buena fuente de proteínas, ácidos grasos, micro elementos, calcio, fósforo, entre otros, para los seres humanos no lactantes. A medida que la demanda mundial de alimentos crece, también lo hacen las exigencias y preocupación de los consumidores por la calidad e inocuidad de los alimentos. Ante evidencias de posibles patologías asociadas al consumo de leche, han aparecido detractores de la industria láctea que explotan estos argumentos. Durante los últimos 20 años se han realizado investigaciones sobre el riesgo asociado al consumo de leche y se ha llegado a determinar la existencia de variantes genéticas de proteínas lácteas específicas, capaces de causar patologías que van desde la intolerancia a la leche y la intensificación de los síntomas asociados, hasta la correlación con diabetes mellitus tipo 1, enfermedad cardiaca isquémica y alergias alimentarias. La determinación de las variantes genéticas de las caseínas y la asociación del alelo A1 de beta-caseína con problemas de salud humana, representan una oportunidad, tanto para los productores primarios de seleccionar adecuadamente a las hembras lecheras de reposición, reteniendo aquellas vacas que porten los alelos deseables; como para la industria, para la obtención de productos lácteos más seguros. De esta manera, la cadena láctea, desde los productores hasta los consumidores, se verán favorecidos.

Palabras clave: caseínas, beta-caseína, kapa-caseína, variantes genéticas, beta casomorfina 7.

\section{INTRODUCCIÓN}

La leche es la fuente más importante de energía, proteínas, vitaminas, además de otros macro y micro nutrientes para los mamíferos luego del nacimiento y durante la primera fase de la vida. En el caso de los humanos, la leche junto a los productos lácteos elabora- dos a partir de ésta constituyen una fuente de energía, ácidos grasos esenciales, proteínas y micronutrientes de alta calidad para todas las edades, en particular los niños (Fernández et al., 2015).

En la dieta de la población humana se incluye leche de una gran variedad de especies animales. Sin embargo, la más consumida en el mundo es la de los bovinos. 
Según la Organización de las Naciones Unidas para la Alimentación y la Agricultura (OECD-FAO, 2016), unos 6.000 millones de personas en todo el mundo consumen leche de vaca, y si bien su consumo es variable, condicionado por aspectos socioculturales y determinado por el nivel de ingresos de la población, FAO proyecta que tanto el consumo como su producción aumenten a unos 177 millones de toneladas (23\% respecto del volumen actual) para el año 2025.

El valor de la leche no solo está representado por su elevada densidad y diversidad de aporte de nutrientes, sino también por sus positivas propiedades biológicas que contribuyen a proteger de algunas enfermedades como cáncer, hipertensión, hipercolesterolemia, entre otras; y por sus propiedades antimicrobianas, inmunomodulatorias y otras asociadas positivamente a la salud humana (Davoodi et al., 2016). Sin embargo, el consumo de leche bovina, y más específicamente, de la proteína láctea, se ha asociado a la presentación de enfermedades crónicas como la diabetes mellitus tipo 1 (Elliott et al., 1999), enfermedad cardíaca isquémica (McLachlan, 2001) y la exacerbación de trastornos digestivos asociados a la intolerancia a la lactosa o alergias de tipo alimentario (Jianqin et al., 2016).

Esta revisión de la literatura aborda aspectos relativos a la producción de leche y rendimiento de la proteína láctea bovina, en particular a la beta-caseína y sus variantes genéticas principales, y el impacto de estas variantes sobre la producción primaria, la calidad tecnológica de la leche y la salud humana.

\section{Proteínas de la leche bovina}

En la fracción proteica de la leche bovina existen dos grandes grupos de proteínas. El grupo cuantitativamente más importante, las caseínas, constituyen el $80 \%$ de las proteínas de la leche. Son las que coagulan para formar la cuajada que finalmente terminará convertida en queso y tienen gran importancia desde un punto de vista nutricional, por su aporte de aminoácidos esenciales, calcio y fósforo. Otras, son las proteínas del suero, proteínas solubles como las albuminas, inmunoglobulinas y lactoglobulinas, siendo las más importantes la alfalactoglobulina y la betalactoglobulinas (Farrell et al., 2004).

Dentro del grupo de las caseínas de la leche bovina se han descrito cuatro tipos distintos: la alfa-caseína S1, alfa-caseína S2, la beta-caseína y la kapa-caseína. Solo la beta-caseína representa alrededor del 30 a 35\% de la caseína total de la leche bovina (Farrell et al., 2004; Brook-Taylor et al., 2017). Las caseínas tienen implicancia directa en la producción primaria de leche, en el procesamiento industrial y en la salud pública.

Cada uno de los tipos de caseínas mencionados más arriba presenta modificaciones en su estructura primaria debido a sustitución o eliminación de uno o más aminoácidos de las cadenas peptídicas de la proteína, como resultado de mutaciones, generando variantes genéticas. Cuando hay más de una variante estructural se habla de polimorfismos o proteínas polimórficas.

\section{Polimorfismo genético de la beta-caseína bovina}

Las variantes genéticas o polimorfismos, son representados por alelos identificados con una letra, normalmente "A" y "B", "A1" y "A2" para el caso los alelos más comunes y de mayor importancia en la beta-caseína (Farrell et al., 2004). Estas variantes genéticas afectan la estructura de las proteínas lácteas generando cambios en las características de estas proteínas y afectan la producción de leche, de sus componentes (Hallén et al., 2008; Heck et al., 2009; Bonfatti et al., 2010; Huang et al., 2012) y las características tecnológicas o aptitud para su uso industrial (Wedholm et al., 2006, Vallas et al., 2012). Además, se ha relacionado el consumo de leche que contiene un tipo específico de beta-caseína, la A1, con enfermedades crónicas como la diabetes mellitus tipo 1, enfermedad cardíaca isquémica y alergias alimentarias (Jianqin et al., 2016).

El gen que codifica la síntesis de beta-caseína se denomina CSN2 y se localiza en el cromosoma 6 del genoma bovino, junto a los demás genes codificantes de caseínas (Ferretti et al., 1990). Para el gen CSN2 se han descrito 13 variantes alélicas de las cuales el alelo $\mathrm{A} 1 \mathrm{y}$ el alelo A2 son las más comunes (Farrell et al., 2004). El alelo $\mathrm{A} 2$ representa el gen original del género Bos. Este gen codifica para la forma alélica A2 de la beta caseína y está presente en la leche de muchos mamíferos, tales como humanos, ovinos, caprinos, además del bovino (Ng-Kwai-Hang y Grosclaude, 2003). Por otra parte, el alelo A1 es una mutación exclusiva en poblaciones de Bos taurus y se encuentra ampliamente distribuida en Europa y América, donde es posible encontrarla en muchas razas lecheras con una amplia diferencia en la frecuencia génica.

Desde un punto de vista bioquímico las moléculas de la proteína beta-caseína láctea se componen de 209 aminoácidos y la diferencia entre las variantes A1 y A2 es tan sólo en un aminoácido en la posición 67 de la cadena peptídica. La variante A1 contiene histidina y la variante A2 contiene prolina (Figura 1). Esta diferencia es la consecuencia de un cambio de nucleótido, que causa el cambio en la estructura de la proteína. El codón original CCT (citosina-citosina-timina) que forma el aminoácido prolina, en la variante A1, se modifica a CAT (citosina-adenina-timina) que codifica la formación de histidina en la posición 67 de la cadena polipeptídica de la beta-caseína (Oleński et al., 2012).

\section{Beta-caseína y composición de la leche bovina}

La producción y concentración de leche bovina es influenciada por varios factores asociados a la vaca o 


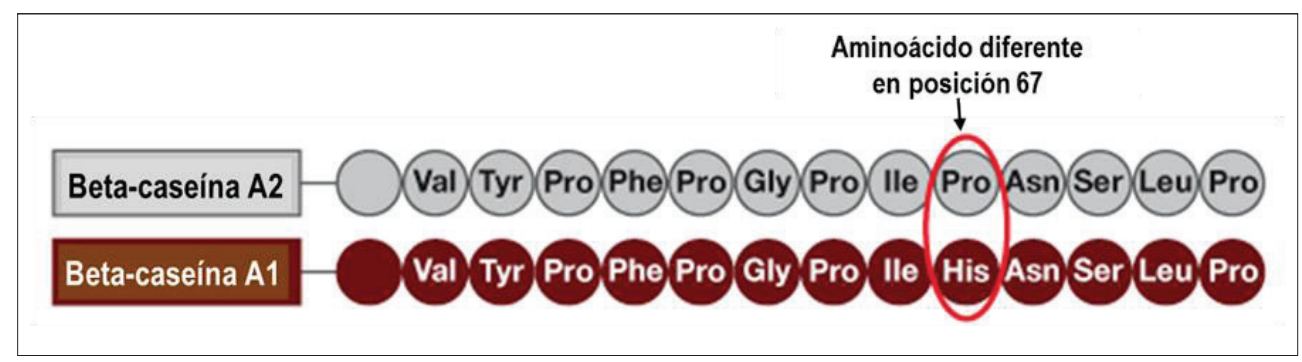

Figura 1. Diferencia en la estructura de la beta-caseína A1 y A2.

Figure 1. Difference in the structure of beta-casein A1 and A2.

al ambiente, entre ellos destacan: la raza, la edad (número de partos), la época del año, la etapa de lactancia, la alimentación y el polimorfismo genético de las proteínas (Huang et al., 2012). Las variantes alélicas de las distintas proteínas de la leche tienen alta influencia en la producción y composición de la leche bovina afectando la concentración total de proteína láctea y la proporción de otras caseínas y proteínas del suero (Heck et al., 2009, Bonfatti et al., 2010).

El polimorfismo genético de las proteínas lácteas ha sido asociado a la composición, características de producción y las propiedades tecnológicas de la leche bovina (Wedholm et al., 2006; Huang et al., 2012). Se ha determinado que las variantes genéticas explican una parte considerable de la variación genética asociada a la composición y características de la proteína láctea (Heck et al., 2009), por lo que deberían ser consideradas en la implementación de programas de selección para la mejora de los rebaños y de la materia prima para la industria láctea.

Como ya se mencionó, las cuatro caseínas se ubican en el mismo locus en el cromosoma 6 del genoma bovino (Ferretti et al., 1990) y la variación de un gen está aparentemente relacionada a la expresión de otros genes dentro del mismo locus (Huang et al., 2012). Esta asociación estrecha desde el punto de vista de ubicación física de los genes, y además de su relación funcional, determinan efectos combinados en la cantidad y proporcionalidad de la síntesis de distintas caseínas (Heck et al., 2009; Bonfatti et al., 2010).

Hallén et al. (2008) y luego Bonfatti et al. (2010), estudiaron el efecto de los genotipos para dos caseínas, beta y kapa, y de betalactoglobulina en la concentración de proteína láctea. Estos estudios confirman que para el genotipo kapa-caseína el alelo B tiende a aumentar la proporción de kapa-caseína y la concentración total de proteína en la leche, mientras que el efecto de betalactoglobulina genotipo BB conduce a un aumento en la concentración de proteína, caseína total y disminución de la betalactoglobulina. Mientras que beta-caseína muestra diferencias en la concentra- ción de proteína total en favor A2 cuando se encuentra asociada a kapa-caseina B, lo que aumenta la concentración total de proteínas y la concentración de betacaseína y kapa-caseína en la leche.

En la misma línea de investigación Heck et al. (2009) determinaron la asociación entre el polimorfismo genético para beta-caseína y características de producción. El alelo A2 demostró tener efectos positivos sobre la producción láctea, proteína y grasa total $(\mathrm{kg})$ y en el porcentaje de proteína, elevando la calidad composicional de la leche y su aptitud para uso industrial.

Estos resultados muestran posibilidades reales de incrementar la producción de leche y la concentración de proteína láctea por la vía de selección de hembras reproductoras utilizando como criterio de selección las variantes genéticas mencionadas. La implementación de este tipo de esquemas de selección impacta no sólo sobre la producción de los rebaños, sino también a nivel industrial, ya que modifica favorablemente las características tecnológicas de la leche y sus posibilidades para ser utilizada en la elaboración de productos lácteos como el queso.

\section{Beta-caseína y aptitud quesera de la leche}

La composición proteica explica el valor nutricional de la leche y también sus propiedades para la industria. Diversos autores han asociado estas características al polimorfismo genético de las proteínas de la leche (Wedholm et al., 2006; Heck et al., 2009; Nilsen et al., 2009; Huang et al., 2012; Vallas et al., 2012).

En términos generales, se sabe que el rendimiento quesero aumenta a medida que aumenta la concentración de caseínas y que el tiempo de coagulación de la leche y la firmeza del cuajo dependen de la composición de caseínas (Heck et al., 2009; Vallas et al., 2012). La cantidad y calidad del queso obtenido, no sólo por litro de leche, sino también por kilo de proteína láctea, dependen de las propiedades de coagulación de la leche (Wedholm et al., 2006), lo que resulta de gran importancia para el resultado económico de la industria 
(Cassandro et al., 2016). Así, la selección de la materia prima por medio de marcadores específicos para detectar propiedades de coagulación y rendimiento quesero constituiría una ventaja económica para la industria. Adicionalmente, la diferencia estructural entre las dos variantes de la beta-caseína, sustitución de prolina (A2) por histidina (A1) en la posición 67 de la cadena aminoacídica, conduce a diferencias en las características de la leche que influyen en la elaboración de productos lácteos, y además en posibles efectos sobre la salud de los consumidores (Nilsen et al., 2009).

Según Wedholm et al. (2006), las propiedades de la leche para la elaboración de quesos pueden ser mejoradas al aumentar la concentración de caseína alfa S1, beta-caseína y kapa-caseína, con una alta concentración de kapa-caseína en relación a la caseína total y, además, con baja concentración de betalactoglobulina. En el mismo trabajo se demostró que leche con una mayor proporción de beta-caseína o enriquecida con beta-caseína en polvo mejora la firmeza de la cuajada. En esta misma línea, Vallas et al. (2012) establecieron que la asociación entre beta-caseína y kapa-caseína es capaz de influir no sólo en la producción y composición de la leche, sino además, sobre el rendimiento quesero y la firmeza de la cuajada. Estos autores asociaron el alelo I de betacaseína (variante del alelo A2) y la kapa-caseína alelo B con un aumento en el porcentaje de proteína, mejor capacidad para coagular y mayor firmeza de la cuajada.

Dado que el consumo de beta-caseína $\mathrm{A} 1$ ha sido asociado, de forma aún no claramente establecida a la presentación de ciertas patologías crónicas y alergias alimentarias, y que además, la leche proveniente de animales homocigotos A2 posee mayor contenido proteico y mejores características para la elaboración de queso, sería apropiado por parte de la industria, adoptar medidas que incentiven el aumento de la leche con alto contenido de beta-caseína del tipo A2, como una forma de mejorar la calidad de la materia prima, y a la vez, reducir el riesgo de presentación de patologías en los consumidores.

\section{Consumo de beta-caseína y riesgos para la salud humana}

El consumo mundial de alimentos de origen animal, incluyendo la leche, ha experimentado importantes incrementos, particularmente en países con economías emergentes (Turk, 2016). El aumento en el consumo de leche en el mundo ha traído como consecuencia el aumento en el riesgo de presentación y empeoramiento de síntomas de algunos problemas de salud asociados a su ingesta. A partir de la década de 1990 diversos autores (Elliot et al., 1999; McLachlan, 2001; Sun et al., 2003) han relacionado el consumo de leche, y específicamente la variante genética A1 de beta-caseína, a problemas de salud que van desde la disfunción gastrointestinal a desórdenes inmunes/inflamatorios, incluso enfermedades de tipo crónico. Los efectos nocivos del consumo de leche se han atribuido a un grupo de péptidos presentes en la leche y derivados de la proteólisis de la beta-caseína A1. En particular destaca el péptido beta casomorfina 7 (BCM-7), al que se atribuyen efectos pro inflamatorios y afinidad por los receptores a morfina (Jianqin et al., 2016).

La composición aminoacídica de la beta-caseína es consecuencia del polimorfismo de los genes que la codifican (Farell et al., 2004). La variante A1 de la betacaseína es más fácilmente hidrolizable por las enzimas digestivas, debido a la débil unión entre histidina e isoleucina (Figura 2), dando como resultado la liberación del péptido BCM-7. Todas las variantes derivadas de A1, que tienen la sustitución histidina por prolina en la posición 67 , tienen el potencial de generar estos péptidos a consecuencia de su digestión. No así, las que derivan

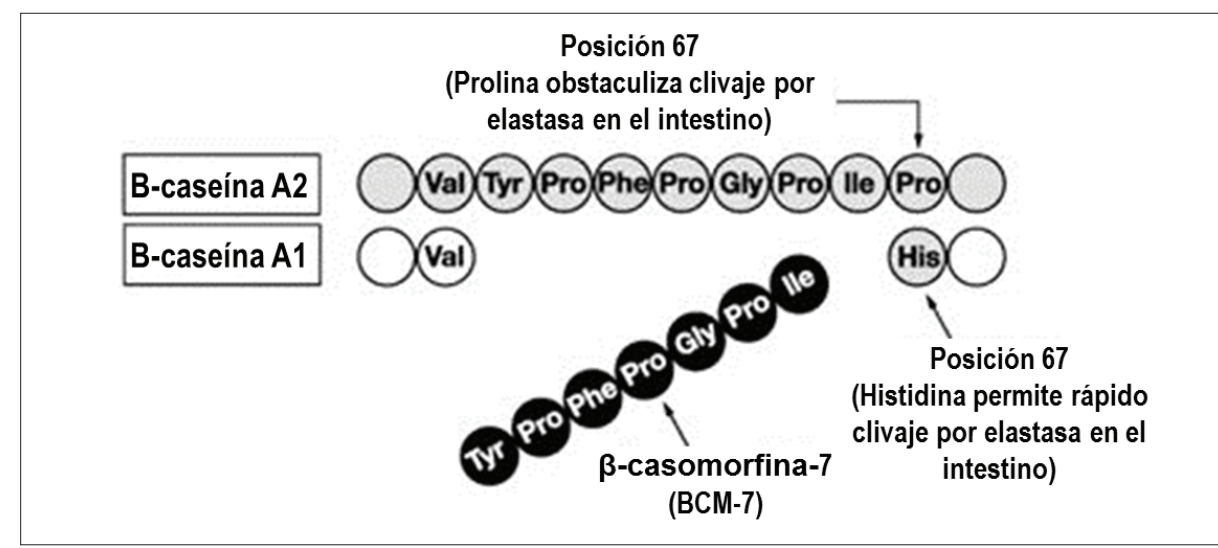

Figura 2. Diferencia de estructura entre beta-caseína A1 y A2 y liberación del péptido BCM-7 (Chia et al., 2017).

Figure 2. Difference in the structure between beta-casein A1 and A2 and liberation of BCM-7 peptide (Chia et al., 2017). 
de la variante A2 (Jianqin et al., 2016), en que la prolina está más firmemente unida a la isoleucina, lo que impediría la liberación del péptido BCM-7. Se ha establecido que la separación de este péptido ocurre por acción de enzimas que actúan en el intestino delgado, pero no por la pepsina en el estómago (Brooke-Taylor et al., 2017).

\section{Enfermedades crónicas asociadas al consumo de beta-caseína $\mathrm{A1}$}

El consumo de beta-caseína A1 en seres humanos ha sido asociada principalmente a alteraciones en la función intestinal (Jianqin et al., 2016) y a dos enfermedades crónicas, la diabetes mellitus tipo 1 en infantes (Chia et al., 2017; Elliott et al., 1999) y la enfermedad cardíaca isquémica en adultos (McLachlan, 2001).

Respecto de la disfunción gastrointestinal, en una revisión hecha por Brooke-Taylor et al. (2017) en que se compararon efectos de ambos tipos de beta caseína, se concluye que el opioide BCM-7 derivado de la forma A1 (pero no de A2) se asocia, tanto en animales como en humanos, con un enlentecimiento del tránsito digestivo y, por lo tanto, con mayor tiempo de tránsito, lo que genera un aumento de procesos fermentativos y molestias gastrointestinales. Además, se han encontrado evidencias de efectos proinflamatorios por la presencia de la BCM-7, aunque los autores no aclaran si este efecto es del opioide per se, o como resultado del tránsito más lento que estaría afectando otros procesos biológicos.

La relación entre las enfermedades del corazón y la beta-caseína A1 ha sido objeto de estudio en modelos animales. Tailford et al. (2003) reportaron un estudio en el que utilizaron conejos a los que se había provocado lesiones aórticas y que recibían dietas con alto contenido de beta-caseína A1 o beta-caseína A2. Los conejos alimentados con beta-caseína A1 mostraron mayor proporción de la superficie aórtica cubierta por estrías grasas, y tenían un mayor espesor de lesiones del arco aórtico que los alimentados con beta-caseína A2. Los que fueron alimentados con más beta-caseína A2 y menor proporción de beta-caseína A1, tenían menos signos de daño cardiovascular, mientras que los animales alimentados con A2 no mostraron daño a las arterias, e incluso, un daño reducido después de la ingesta de colesterol. La conclusión de este trabajo, indicando que la leche de vacas con genotipo A1-A1 presenta mayores riesgos para la población, ha sido impugnada (Mann y Skeaff, 2003) debido a que el conejo es una especie particularmente sensible a desarrollar en forma bastante rápida este tipo de lesiones, y aunque los resultados permitirían adelantar una hipótesis de trabajo, debería haber suficiente evidencia con humanos antes de concluir sobre el efecto aterogénico de la forma A1 o el efecto protector de A2.

Estudios prospectivos con humanos en búsqueda de poder asociar el consumo de leche con el aumento de patologías, muestran por ejemplo, que las personas que originalmente vivían en África oriental, prácticamente no tenían enfermedades cardiovasculares a pesar del consumo de una dieta rica en leche bovina. Esta leche se obtiene del ganado Cebú, que sólo presenta el alelo A2 de beta-caseína. En los países occidentales que presentan un elevado consumo de leche bovina, predominantemente proveniente de la raza Holstein, existe una mayor incidencia de enfermedades cardiovasculares que en las naciones con bajo consumo de leche (McLachlan, 2001). Este autor mostró además una elevada correlación entre el consumo estimado de proteína del tipo A1 y mortalidad cardiovascular para el caso de Alemania.

Elliott et al. (1999) investigaron la correlación entre el consumo de leche con distintas variantes genéticas para beta-caseína, y la presentación de diabetes mellitus tipo 1 (insulina dependiente) en niños de hasta 14 años de edad de diez países diferentes. Este estudio permitió determinar que el consumo de proteína total de la leche no estaría asociado a la presentación de diabetes mellitus tipo 1 . Sin embargo, el consumo de betacaseína A1 sí mostró una alta correlación. Los autores concluyen que el rol inmunosupresor de la BCM-7 jugaría un papel fundamental en la presentación de la enfermedad. Laugesen y Elliott (2003) realizaron un estudio basándose en la casuística de enfermedad cardíaca isquémica y diabetes mellitus tipo 1 en 20 países del norte de Europa en un periodo de 20 años. Asociaron el consumo per cápita de leche, crema y queso a una ingesta de beta-caseína A1, y encontraron asociaciones significativas entre el consumo de esa variante genética de beta-caseína y las enfermedades mencionadas. Sin embargo, no encontraron asociaciones benéficas relacionadas a la variante $\mathrm{A} 2$.

Pese a la existencia de evidencia de asociación entre éstas y otras patologías con el consumo de leche con beta-caseína A1, aún no existe una relación de causalidad plenamente establecida, y el mecanismo exacto en que la beta-caseína favorece la presentación de estas patologías no está del todo claro, siendo éste un campo de estudio en pleno desarrollo.

\section{Alergia alimentaria infantil}

Según los datos presentados por el National Institute of Allergy and Infectious Diseases, NIAID (Boyce et al., 2010), la presentación de alergias de tipo alimentario es baja, y varía entre un 3\% y un 8\% de la casuística asociada a la presentación de reacciones adversas a alimentos entre los menores de 18 años. Este tipo de alergias incluye, además de las reacciones a los posibles alérgenos de la leche, otros 170 alimentos como el huevo, soya, maní, nueces, trigo, pescados y mariscos. Según los datos del mismo organismo, el porcentaje de presentación de alergias alimentarias iría en incre- 
mento a nivel internacional, y está asociada a diversos factores como la edad, el origen racial, el grado de urbanización y los ingresos familiares. La alergia asociada al consumo de leche es más común (pero no excluyente) entre los menores de 2 años, de origen africano o asiático, que habitan en centros urbanos, y la patología es más frecuentemente diagnosticada a medida que el ingreso familiar aumenta.

En Chile no existen estudios de prevalencia de alergia asociada al consumo de leche en niños ni en adultos. Sin embargo, el Ministerio de Salud (Ministerio de Salud de Chile, 2012), incluye a la Alergia a la Proteína de Leche de Vaca (APLV) en sus protocolos clínicos de diagnóstico, y su objetivo es evitar errores en el diagnóstico, dado el alto impacto médico, social y económico en el paciente afectado y su familia. Ya que el tratamiento generalmente considera evitar en forma estricta la proteína de leche de vaca, esta enfermedad tiene una carga económica importante. Además, las formas graves de APLV pueden amenazar la vida del paciente, particularmente en formas inmediatas (mediadas por IgE) que conducen a shock anafiláctico. Según la "Guía Clínica: Alergia a Proteína de Leche de Vaca" (Ministerio de Salud de Chile, 2012), los factores de riesgo principales para desarrollar alergias a alimentos son la edad del paciente, su historia familiar de atopia y enfermedades alérgicas y la co-morbilidad personal de enfermedades alérgicas, particularmente dermatitis atópica.

Los lactantes son más susceptibles a presentar patologías asociadas al consumo de proteínas, ya que sus barreras anatómicas y funcionales (acidez gástrica, enzimas intestinales y glicocálix) y barreras inmunológicas (IgA secretora), no han alcanzado su desarrollo pleno, permitiendo el paso de antígenos alimentarios al torrente sanguíneo. Es decir, existe menor capacidad de "tolerancia" del sistema inmune intestinal (Vitaliti et al., 2012). Un elemento fundamental en el desarrollo del mecanismo adaptativo inmunológico es el microbioma intestinal y la presencia de IgA intestinal. Aquí juega un rol primordial la alimentación con leche materna y la alimentación de la madre lactante. La betacaseína de la leche humana es del tipo A2, por lo cual la lactancia materna temprana y prolongada evita al lactante la exposición temprana a la beta-caseína A1, aunque madres que consumen leche bovina pueden transferir BCM-7 a través de su leche al infante (Chia et al., 2017). Se ha reportado que la eliminación de leche de vaca en la alimentación de madres lactantes deriva en niveles reducidos de IgA específica en la leche materna, lo que se asocia con el desarrollo de alergia a la leche de vaca en infantes (Järvinen et al., 2014).

\section{CONCLUSIONES}

El incremento en el consumo de leche en el mundo supone desafíos para la producción primaria, industria y la salud pública. La búsqueda de materias primas más seguras y productos lácteos más inocuos, plantea el desafío de seleccionar reproductores que porten aquellos alelos que demuestren ser más seguros para los consumidores de leche, y a la vez, que ofrezcan mejoras en la producción y los componentes de la leche, de forma tal, que toda la cadena de suministro lácteo se beneficie de decisiones conscientes y responsables.

Pese a que existe evidencia para asociar el consumo de leche con beta-caseína A1 a diversas alteraciones de salud, tales como molestias gastrointestinales, diabetes mellitus tipo 1 , enfermedad cardíaca isquémica y alergias alimentarias, aún no existe una relación de causalidad plenamente establecida, y el mecanismo exacto en que la beta-caseína y la BCM-7 favorecen la presentación de estas patologías aún está bajo estudio. Se requiere más investigación para esclarecer los mecanismos involucrados, y también sobre el rol protector del consumo de la proteína A2.

\section{REFERENCIAS}

Bonfatti, V., Di Martino, G., Cecchinato, A., Vicario, D., Carnier, P., 2010. Effects of $\beta$ - $\kappa$-casein (CSN2-CSN3) haplotypes and $\beta$-lactoglobulin (BLG) genotypes on milk production traits and detailed protein composition of individual milk of Simmental cows. Journal of Dairy Science 93(8), 3797-3808. https://doi.org/10.3168/jds.2009-2778

Boyce, J.A., Assa'ad, A., Burks, A.,W., Jones, S.M., Sampson, H.A., Wood, R.A., Plaut, M., Cooper, S.F., Fenton, M.J., Arshad, S.H., Bahna, S.L., Beck, L.A., Byrd-Bredbenner, C., Camargo, C.A., Eichenfield, L., Furuta, G.T., Hanifin, J.M., Jones, C., Kraft, M., Levy, B.D., Lieberman, P., Luccioli, S., McCall, K.M., Schneider, L.C., Simon, R.A., Simons, F.E., Teach, S.J., Yawn, B.P., Schwaninger, J.M., 2010. Guidelines for the diagnosis and management of food allergy in the United States: report of the NIAID-sponsored expert panel. Journal of Allergy and Clinical Immunology 126 (6), S1S58. https://doi.org/10.1016/j.jaci.2010.10.007

Brook-Taylor, S., Dwyer, K., Woodford, K., Kost, N., 2017. Systematic review of the gastrointestinal effects of A1 compared with A2 $\beta$-casein. Advances in Nutrition 8(5), 739748. https://doi.org/10.3945/an.116.013953

Cassandro, M., Pretto, D., Lopez-Villalobos, N., De Marchi, M., Penasa, M., 2016. Estimation of economic values for milk coagulation properties in Italian Holstein-Friesian cattle. Journal of Dairy Science 99(8), 6619-6626. https:// doi.org/10.3168/jds.2015-10228

Chia, J.S.J., McRae, J.L., Kukuljan, S., Woodford, K., Elliott, R.B., Swinburn, B., Dwyer, K.M., 2017. A1 beta-casein milk protein and other environmental pre-disposing factors for type 1 diabetes. Nutrition \& Diabetes 7, e274. https://doi.org/10.1038/nutd.2017.16

Davoodi, S.H., Shahbazi, R., Esmaeili, S., Sohrabvandi, S., Mortazavian, A., Jazayeri, S., Taslimi, A., 2016. Health-related aspects of milk proteins. Iranian Journal of Pharmaceutical Research 15(3), 573-591.

Elliott, R.B., Harris, D.P., Hill, J.P., Bibby, N.J., Wasmuth, H.E., 1999. Type I (insulin-dependent) diabetes mellitus 
and cow milk: casein variant consumption. Diabetologia 42(3), 292-296. https://doi.org/10.1007/ s001250051153

Farrell Jr., H.M., Jimenez-Flores, R., Bleck, G.T., Brown, E.M., Butler, J.E., Creamer, L.K., Hicks, C.L., Hollar, C.M., NgKwai-Hang, K.F., Swaisgood, H.E., 2004. Nomenclature of the proteins of cows' milk-sixth revision. Journal of Dairy Science 87(6), 1641-1674. https://doi.org/10.3168/ jds.S0022-0302(04)73319-6

Fernández, E., Martínez, J.A., Martínez, V., Moreno, J.M., Collado, L.R., Hernández, M., Morán, F.J., 2015. Documento de consenso: importancia nutricional y metabólica de la leche. Nutrición Hospitalaria 31(1), 92-101. http:// dx.doi.org/10.3305/nh.2015.31.1.8253

Ferretti, L., Leone, P., Sgaramella, V., 1990. Long range restriction analysis of the bovine casein genes. Nucleic Acids Research 18(23), 6829-6833. https://doi.org/10.1093/ nar/18.23.6829

Hallén, E., Wedholm, A., Andrén, A., Lundén, A., 2008. Effect of $\beta$-casein, $\kappa$-casein and $\beta$-lactoglobulin genotypes on concentration of milk protein variants. Journal of Animal Breeding and Genetics 125(2), 119-129. https:// doi.org/10.1111/j.1439-0388.2007.00706.x

Heck, J., Schennink, A., Van Valenberg, H., Bovenhuis, H., Visker, M., Van Arendonk, J., Van Hooijdonk, A., 2009. Effects of milk protein variants on the protein composition of bovine milk. Journal of Dairy Science 92(3), 1192-1202. https://doi.org/10.3168/jds.2008-1208

Huang, W., Peñagaricano, F., Ahmad, K.R., Lucey, J.A., Weigel, K.A., Khatib, H., 2012. Association between milk protein gene variants and protein composition traits in dairy cattle. Journal of Dairy Science 95(1), 440-449. https:// doi.org/10.3168/jds.2011-4757

Järvinen, K.M., Westfall, J.E., Seppo, M.S., James, A.K., Tsuang, A.J., Feustel, P.J., Sampson, H.A., Berin, C., 2014. Role of maternal elimination diets and human milk IgA in the development of cow's milk allergy in the infants. Clinical \& Experimental Allergy 44(1), 69-78. https://doi. org/10.1111/cea.12228

Jianqin, S., Leiming, X., Lu, X., Yelland, G., Ni, J., Clarke, A.J., 2016. Effects of milk containing only A2 beta casein versus milk containing both $\mathrm{A} 1$ and $\mathrm{A} 2$ beta casein proteins on gastrointestinal physiology, symptoms of discomfort, and cognitive behavior of people with self-reported intolerance to traditional cows' milk. Nutrition Journal 15, 35. https://doi.org/10.1186/s12937-016-0147-z

Laugesen, M., Elliott, R., 2003. Ischaemic heart disease, type 1 diabetes, and cow milk A1 beta-casein. New Zealand Medical Journal 116(1168), 1-19.

Mann, J., Skeaff, M., 2003. $\beta$-casein variants and atherosclerosis-claims are premature. Atherosclerosis 170(1), 11-
12. https://doi.org/10.1016/S0021-9150(03)00230-2

McLachlan, C., 2001. $\beta$-casein A1, ischaemic heart disease mortality, and other illnesses. Medical Hypotheses 56(2), 262-272. https://doi.org/10.1054/mehy.2000.1265

Ministerio de Salud de Chile, 2012. Guía clínica: alergia a proteína de leche de vaca. Subsecretaría de Salud Pública, Ministerio de Salud de Chile, Santiago, Chile.

Ng-Kwai-Hang, K.F., Grosclaude, F., 2003. Genetic polymorphism of milk proteins, in: Fox, P.F., McSweeney, P.L.H. (Eds.), Advanced dairy chemistry: volume 1: proteins. Springer, Boston, MA, pp. 739-816.

Nilsen, H., Olsen, H., Hayes, B., Sehested, E., Svendsen, M., Nome, T., Meuwissen, T., Lien, S., 2009. Casein haplotypes and their association with milk production traits in Norwegian Red cattle. Genetics Selection Evolution 41(1), 24. https://doi.org/10.1186/1297-9686-41-24

Organisation for Economic Co-operation and DevelopmentFood and Agriculture Organization of the United Nations (OECD-FAO), 2016. OECD-FAO Agricultural Outlook 2016-2025. OECD Publishing, Paris.

Oleński, K., Cieślińska, A., Suchocki, T., Szyda, J., Kamiński, S., 2012. Polymorphism in coding and regulatory sequences of beta-casein gene is associated with milk production traits in Holstein-Friesian cattle. Animal Science Papers and Reports 30(1), 5-12.

Sun, Z., Zhang, Z., Wang, X., Cade, R., Elmir, Z., Fregly, M., 2003. Relation of $\beta$-casomorphin to apnea in sudden infant death syndrome. Peptides 24(6), 937-943. https://doi. org/10.1016/S0196-9781(03)00156-6

Tailford, K.A., Berry, C.L., Thomas, A.C., Campbell, J.H., 2003. A casein variant in cow's milk is atherogenic. Atherosclerosis 170(1), 13-19. https://doi.org/10.1016/ S0021-9150(03)00131-X

Turk, J., 2016. Meeting projected food demands by 2050: understanding and enhancing the role of grazing ruminants. Journal of Animal Science 94(6), 53-62. https:// doi.org/10.2527/jas.2016-0547

Vallas, M., Kaart, T., Värv, S., Pärna, K., Jõudu, I., Viinalass, H., Pärna, E., 2012. Composite $\beta$ - $\kappa$-casein genotypes and their effect on composition and coagulation of milk from Estonian Holstein cows. Journal of Dairy Science 95(11), 6760-6769. https://doi.org/10.3168/jds.2012-5495

Vitaliti, G., Cimino, C., Coco, A., Praticò, A.D., Lionetti, E., 2012. The immunopathogenesis of cow's milk protein allergy (CMPA). Italian Journal of Pediatrics 38(1), 35. https:// doi.org/10.1186/1824-7288-38-35

Wedholm, A., Larsen, L.B., Lindmark-Mansson, H., Karlsson, A.H., Andrén, A., 2006. Effect of protein composition on the cheese-making properties of milk from individual dairy cows. Journal of Dairy Science 89(9), 3296-3305. https://doi.org/10.3168/jds.S0022-0302(06)72366-9 
